\title{
A CLASS OF TOPOLOGICAL SPACES
}

\section{EDWIN HEWITT}

1. Introduction. It is a classical theorem of set-theoretical topology that a one-to-one continuous mapping of a bicompact Hausdorff space onto a Hausdorff space is a homeomorphism. ${ }^{1}$ Stated in somewhat different terms, this theorem asserts that any bicompact Hausdorff topology on a given set $E$ is a minimal Hausdorff topology. If $B$ is the family of open sets in this topology, then no proper subfamily of $\mathcal{B}$ can be the family of open sets for a Hausdorff topology on $E$. When this phenomenon is observed, a number of questions immediately present themselves:

(1) Under what conditions will a minimal Hausdorff space be bicompact?

(2) Are there any minimal Hausdorff spaces which are not bicompact?

(3) Is there any simple way of characterizing those topological spaces which have one-to-one continuous images which are bicompact Hausdorff spaces?

Question (1) was answered completely by Katětov [6, p. 40], who proved that a Hausdorff space is bicompact if and only if it is minimal and satisfies the Urysohn separation axiom (that is, every pair of distinct points possess neighborhoods whose closures are disjoint).

Question (2) can be answered in the affirmative by modifying a space constructed by Urysohn (see [2, p. 22]). Let the space $X_{0}$ be defined as the set of all points $(x, y)$ in the Euclidean plane such that $0<x^{2}+y^{2} \leqq 1$, together with two adjoined points $p^{+}$and $p^{-}$. Let neighborhoods of all points $(x, y)$ in $X_{0}$ be the usual Euclidean neighborhoods; let $U_{n}\left(p^{+}\right)$be $p^{+} \cup E\left[(x, y), 0<x^{2}+y^{2}<1 / n, y>0\right]$; and let $U_{n}\left(p^{-}\right)$be $p^{-} \cup E\left[(x, y), 0<x^{2}+y^{2}<1 / n, y<0\right]$. As the index $n$ assumes all positive integral values, the neighborhoods $U_{n}\left(p^{+}\right)$and $U_{n}\left(p^{-}\right)$describe a complete family of neighborhoods for $p^{+}$and $p^{-}$. It is obvious that, under this definition, $X_{0}$ forms a Hausdorff space which fails to satisfy the Urysohn separation axiom. It is easy to prove, moreover, that $X_{0}$ is a minimal Hausdorff space. Katětov [6,

Presented to the Society, April 17, 1948; received by the editors March 22, 1948.

${ }^{1}$ See, for example [1, p. 95, Satz III]. It is interesting to observe that this result was stated in 1893 by Jordan for bounded closed subsets of $n$-dimensional Euclidean space (see [5, p. 53]). Numbers in brackets refer to the references cited at the end of the paper. 
p. 40] has proved that a Hausdorff space is minimal if and only if it is semi-regular (that is, sets of the form $A^{-1-1}$ form a basis for the open sets) and $H$-closed (that is, closed in any Hausdorff space which contains it as a subspace). $H$-closure is equivalent to the assertion that every covering by a family of open sets admits a finite subfamily whose closures form a covering $\left[1\right.$, p. 90, Satz X]. Since the space $X_{0}$ is obviously semi-regular and $H$-closed, it must be minimal. It is obviously not bicompact, however, being irregular. One may observe also that the set of points $\{(1 / n, 0)\}_{n=1}^{\infty}$ has no limit point.

The purpose of the present note is to answer question (3).

2. Rings of continuous functions. If $X$ is any topological space, we designate the set of all real-valued continuous functions on $X$ by the symbol $\mathfrak{S}(X, R)$ and the set of all bounded continuous real-valued functions on $X$ by the symbol $\mathfrak{C}^{*}(X, R)$. These sets of functions are algebraic rings of a very special type, and have been studied in detail by a number of authors. (See, for example, [7, pp. 453 et. seq. $]$ and $[\mathbf{4}$, passim $]$.) We propose to use properties of these function rings in solving our present problem. In considering rings $\complement^{*}(X, R)$, we may limit ourselves to bicompact Hausdorff spaces, since, to every completely regular space $X$, one may assign a uniquely determined bicompact Hausdorff space $\beta X$ which contains $X$ as a dense subspace and which has the property that $\mathfrak{S}^{*}(\beta X, R)$ is algebraically isomorphic to $\mathbb{S}^{*}(X, R)$. A subring of a ring $\mathfrak{E}(X, R)$ is said to be an analytic subring if it is closed in the uniform topology for the ring $\mathfrak{E}(X, R)$ and contains the constant functions. In comparing topological properties of a bicompact Hausdorff space with algebraic properties of its function ring $\mathbb{S}^{*}(X, R)$, the following facts become evident.

(4) Every analytic subring of $\mathfrak{S}^{*}(X, R)$ is isomorphic to some ring $\mathcal{C}^{*}(Y, R)$, where $Y$ is a continuous image of $X$ which is a (necessarily bicompact) Hausdorff space. Conversely, if $Y$ is a bicompact Hausdorff space which is a continuous image of $X$, then the function ring $\mathfrak{S}^{*}(Y, R)$ is isomorphic to an analytic subring of $\mathfrak{S}^{*}(X, R)$.

(5) If $X$ is any completely regular space, and if $Y$ is a completely regular space which is a continuous image of $X$, then the ring $\mathbb{E}(X, R)$ contains an analytic subring which is isomorphic to the ring $\mathbb{C}(Y, R)$.

(6) If $X$ is a bicompact Hausdorff space, every maximal ideal in $\mathfrak{C}^{*}(X, R)$ is the set of all functions in $\mathfrak{\complement}^{*}(X, R)$ vanishing at some point of $X$.

Statements (4) and (6) were proved by Stone [7, Theorems 81 and $80]$. Statement (5) is obviously true. It may be noted that the converse of (5) is false. As an example, consider the space $R$ consisting of 
the real numbers in their usual topology, and let $\mathfrak{B}$ be the analytic subring of $\mathbb{E}(R, R)$ generated by the constant functions and the function $f(x)=x$. It is clear that $\mathfrak{B}$ is a proper subring of $\mathbb{S}(R, R)$, since it does not contain, for example, the function $e^{x}$. It can readily be shown, using methods developed in [4], that $\mathfrak{P}$ cannot be isomorphic to the ring $\mathfrak{C}(X, R)$ for any topological space $X$. If $\mathfrak{B}$ were isomorphic to such a ring, then one could obtain a space $X$ such that $\mathfrak{B}$ is isomorphic to $\mathfrak{E}(X, R)$ by considering all maximal ideals $\mathfrak{M}$ in $\mathfrak{B}$ such that $\mathfrak{B} / \mathfrak{M}$ is algebraically isomorphic to $R$ and topologizing them in accordance with [4, Theorem 9]. Upon carrying out this process, one simply obtains $R$ in its usual topology, which produces an evident contradiction.

3. Limitation of spaces considered. Since every bicompact Hausdorff space $X$ is normal, it is clear that for distinct points $p$ and $q$ in $X$, there is a function $g \in \mathfrak{S}(X, R)$ such that $g(p)=1$ and $g(q)=0$. This property being preserved upon passage to any stronger topology, we may evidently limit ourselves to spaces having this separation property. On the other hand, it is well known that any space with this property has a one-to-one continuous image which is completely regular. (See for example [4, Theorem 4].) We may thus make a second limitation, and consider only completely regular spaces.

Among completely regular spaces, we single out a certain class, namely, those spaces $X$ whose topology can be completely described in terms of the algebraic structure of the ring $\mathbb{E}(X, R)$. This statement can be rendered more precise. An ideal $\Im$ in $\mathfrak{S}(X, R)$ is said to be real

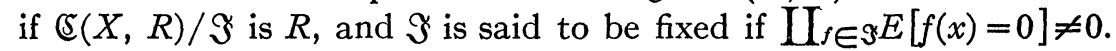
The topology of a completely regular space $X$ can be described in terms of the algebraic structure of $\mathbb{E}(X, R)$ if and only if every real ideal in $\mathfrak{S}(X, R)$ is fixed. We call such spaces $Q$-spaces, and have investigated their properties in [4]. We find it essential in the present discussion to limit our attention to $Q$-spaces.

4. Main theorem. Our technique is to translate a topological property into a property of function rings and then to retranslate this algebraic property into a topological property.

Theorem A. $A Q$-space $X$ has the property that there exists a bicompact Hausdorff space $Y$ which is a one-to-one continuous image of $X$ if and only if the ring $\mathfrak{C}^{*}(X, R)$ contains an analytic subring $\mathfrak{A}$ such that every maximal ideal in $\mathfrak{A}$ can be imbedded in precisely one real ideal of $\mathfrak{S}(X, R)$.

Suppose that $Y$ is a bicompact Hausdorff space which is a one-to- 
one continuous image of $X$ under a mapping $\Phi$. Then the functions $f \Phi(x)$, where $f \in \mathfrak{C}^{*}(Y, R)$, form an analytic subring $\mathfrak{A}$ of $\mathfrak{C}^{*}(X, R)$ which is isomorphic to the ring $\mathcal{C}^{*}(Y, R)$. By remark (6), the points of $Y$ are in one-to-one correspondence with the maximal ideals of $\mathcal{S}^{*}(Y, R)$, and hence of $\mathfrak{A}$. Since $\Phi$ is a one-to-one mapping, it is plain that the functions in any maximal ideal $\mathfrak{M}$ of $\mathfrak{U}$ all vanish at exactly one point of $X$. The ideal $\mathfrak{M}_{p}$ in $\mathfrak{S}(X, R)$ consisting of all functions in $\mathfrak{C}(X, R)$ vanishing at $p$ is the unique extension of $\mathfrak{M}$ to a real ideal in $\mathfrak{E}(X, R)$.

Conversely, suppose that $\mathbb{C}^{*}(X, R)$ contains a subring of the kind specified above. Then the set of all maximal ideals of $\mathfrak{A}$ may be topologized (see [4, Theorem 9]) to form a bicompact Hausdorff space $Y$ which is a continuous image of the space $\beta X$. $\beta X$ is to be considered as the space of all maximal ideals in $\mathfrak{\complement}(X, R)$ (see $[4$, Theorem $46]$ ), and the continuous mapping of $\beta X$ onto $Y$ is obtained by mapping a given maximal ideal of $\beta X$ onto its intersection with $\mathfrak{A} . X$ being a $Q$-space, all of the real ideals in $\mathbb{E}(X, R)$ are fixed, and the hypotheses on $\mathfrak{A}$ make it clear that the mapping just described produces a one-to-one continuous mapping of $X$ onto $Y$.

THEOREM B. A Q-space $X$ has a one-to-one continuous image $Y$ which is a bicompact Hausdorff space if and only if the family $Z(X)$ contains a subfamily $A$ such that: (1) given $p \neq q$ in $X$, there is a set $B \in \mathcal{C}$ containing exactly one of $p$ and $q ; \mathrm{I}_{A \in \mathcal{C} A A}$ is void; (2) any subfamily of $A$ with the finite intersection property has total intersection nonvoid.

We use Theorem A to prove the present theorem, showing that the conditions stated above are equivalent to the existence of a subring $\mathfrak{A}$ of $\mathfrak{C}^{*}(X, R)$ having the properties set forth in Theorem A. Suppose that such a subring exists. Let $\mathfrak{B}$ be the set of functions in $\mathfrak{A}$ which vanish somewhere in $X$. It is plain that $\mathscr{B}$ is precisely the set of functions in $\mathfrak{A}$ not having inverses. The family $\mathcal{A}=\{Z(f)\}_{f \in \mathfrak{B}}$ is a family with the properties required. We may obviously exclude the trivial case in which $\mathfrak{A}$ contains exactly one maximal ideal. Assume that condition (1) fails for points $p$ and $q$ in $X$. Then, the maximal ideal in $\mathfrak{A}$ consisting of all functions in $\mathfrak{A}$ which vanish at $q$ is contained in the distinct maximal real ideals $\mathfrak{M}_{p}$ and $\mathfrak{M}_{q}$ of $\mathfrak{E}(X, R)$, a contradiction. Next, let $\mathcal{F}$ be any subfamily of $\mathcal{A}$ with the finite intersection property. The functions $f$ in $\mathfrak{A}$ such that $Z(f) \in \mathcal{F}$ generate a proper ideal in $\mathfrak{A}$, which is contained in a maximal real ideal of $\mathfrak{S}(X, R)$. Since $X$ is a $Q$-space, it follows that $\mathcal{F}$ has total intersection nonvoid.

${ }^{2}$ Let $Z(f)$ be the set of points where the function $f$ vanishes. Then $Z(X)$ is the family of all $Z(f)$, for $f \in \mathbb{S}(X, R)$. 
The converse is easily established. Let $\mathcal{A}$ be a subfamily of $Z(X)$ of the kind described, and let $\mathfrak{A}$ be the analytic subring of $\mathfrak{E}^{*}(X, R)$ generated by all bounded continuous real-valued functions such that $Z(f) \in \mathcal{C}$. It is clear that every maximal ideal in $\mathfrak{A}$ is contained in precisely one real ideal in $\mathfrak{E}(X, R)$, and this observation completes the present proof.

5. Examples. It is instructive to observe Theorems A and B in relation to certain common spaces.

(7) No completely regular space of cardinal number less than $2^{\text {so }_{0}}$ and devoid of isolated points can have a one-to-one continuous mapping onto a bicompact Hausdorff space. This is obvious from the fact that such a bicompact Hausdorff space would have to be dense in itself, and such spaces are known to have cardinal number not less than $2^{N_{0}}$. (See $[2$, p. 30 , corollaire 1$\left.].\right)^{3}$

(8) Any locally bicompact Hausdorff space $X$ has a one-to-one continuous mapping onto a bicompact Hausdorff space. Let $p$ be any point of $X$, and let new neighborhoods of $p$ be $U(p) \cup F^{\prime}$, where $U(p)$ is a generic neighborhood of $p$ and $F$ is any bicompact subset of $X$. This new topology establishes the desired result.

(9) Let $\left\{X_{\lambda}\right\}_{\lambda \in \Delta}$ be a family of topological spaces each having a one-to-one continuous map onto a bicompact Hausdorff space $Y_{\lambda}$. Then the Cartesian product $P_{\lambda \in \Lambda} X_{\lambda}$ admits an obvious one-to-one continuous mapping onto the bicompact Hausdorff space $P_{\lambda \in \Lambda} Y_{\lambda}$.

We next illustrate Theorem A by examining a simple space.

(10) Let $\Gamma$ be the rational numbers in the closed interval $[0,1]$ in their usual topology. Theorem A and remark (7) imply that any analytic subring $\mathfrak{A}$ of $\mathfrak{C}^{*}(\Gamma, R)$, such that for all $r_{1} \neq r_{2}$ in $\Gamma$ there exists $f \in \mathfrak{A}$ with $f\left(r_{1}\right) \neq f\left(r_{2}\right)$, contains maximal ideals contained in no real ideal of $\mathfrak{C}(\Gamma, R)$. ( $\Gamma$ is a $Q$-space, like every separable metric space.) Let $\mathfrak{B}$ be the analytic subring of $\mathfrak{S}^{*}(\Gamma, R)$ generated by the constants and the function $f(r)=r$. Plainly this ring is isomorphic to $\complement^{*}(I, R)$, where $I$ is the closed interval $[0,1]$. All ideals $\mathfrak{M}_{t}$ in this ring, where $t$ is any irrational number, are contained in no real ideal of $\mathfrak{E}(\Gamma, R)$. Next, let $t$ be any irrational number in $(0,1)$, and let $\phi_{t}$ be that function on $\Gamma$ such that $\phi_{t}(r)=1$ for $r<t$ and $\phi_{t}(r)=0$ for $r>t$. Let $\mathfrak{Q}$ denote the analytic subring of $\mathfrak{C}^{*}(\Gamma, R)$ generated by the functions $\phi_{t}$ and the constant functions. It can then be proved that $\mathfrak{Q}=\complement^{*}(\Gamma, R)$. Let $F$ be any closed subset of $\Gamma$. It is easy to see that $F^{\prime}$ is the union

${ }^{8}$ Katětov has observed (in a letter to the writer) that no countable Hausdorff space dense in itself can be $H$-closed, which is a stronger statement than the present remark, for countable spaces. 
of disjoint open intervals $\left(a_{\lambda}, b_{\lambda}\right)$ in $\Gamma$, where $a_{\lambda}$ and $b_{\lambda}$ can be either rational or irrational. If $a_{\lambda}$ and $b_{\lambda}$ are irrational, let $g_{\lambda}$ be the charactetistic function of $\left(a_{\lambda}, b_{\lambda}\right)$, defined only on $\Gamma$, of course. If $a_{\lambda}$ and $b_{\lambda}$ are rational, let $g_{\lambda}$ be

$$
e^{-\tan ^{2}\left[\pi\left(x-b_{\lambda}\right) /\left(b_{\lambda}-a_{\lambda}\right)+\pi / 2\right]}
$$

in $\left(a_{\lambda}, b_{\lambda}\right)$ and zero elsewhere. If exactly one of $a_{\lambda}$ and $b_{\lambda}$ is rational, say $a_{\lambda}$, let $g_{\lambda}$ be equal to $\left(x-b_{\lambda}\right) /\left(b_{\lambda}-a_{\lambda}\right)+1$ in $\left(a_{\lambda}, b_{\lambda}\right)$ and zero elsewhere. The function $f=\sum_{\lambda} g_{\lambda}$ is clearly in $\mathfrak{Q}$, and has the property that $f=0$ exactly on $F$. If $F_{1}$ and $F_{2}$ are disjoint closed subsets of $\Gamma$, with functions $f_{1}$ and $f_{2}$ as just defined, then $f_{1} /\left(f_{1}+f_{2}\right)$ is a function in $\mathfrak{Q}$ which vanishes on $F_{1}$ and equals 1 throughout $F_{2}$. By a generalized approximation theorem [3], it follows that $\mathfrak{Q}=\mathbb{C}^{*}(\Gamma, R)$. Clearly $\mathfrak{Q}$ fails to meet the requirements of Theorem $A$, since the space of maximal ideals in $\mathfrak{Q}$ can be considered as the space $\beta \Gamma$, which contains $2^{2 \aleph_{0}}$ points.

\section{REFERENCES}

1. P. S. Alexandroff and H. Hopf, Topologie I, Berlin, Springer, 1935.

2. P.S. Alexandroff and P. Urysohn, Mémoire sur les espaces topologiques compacts, Verhandelingèn der Koninklijke Akademie van Wetenschapen te Amsterdam, Afdeeling Natuurkunde, vol. 14 (1929).

3. Edwin Hewitt, Certain generalizations of the Weierstrass approximation theorem, Duke Math. J. vol. 14 (1947) pp. 419-427.

4. - , Rings of real-valued continuous functions. I, Trans. Amer. Math. Soc. vol. 64 (1948) pp. 45-99.

5. C. Jordan, Cours d'analyse, $2 \mathrm{~d}$ ed., vol. 1, Paris, Gauthier-Villars, 1893.

6. Miroslav Katětov, Über H-abgeschlossene und bikompakte Räume. Casopis pro Péstování Matematiky a Fysiky vol. 69 (1940) pp. 36-49.

7. M. H. Stone, Applications of the theory of Boolean rings to general topology, Trans. Amer. Math. Soc. vol. 41 (1937) pp. 375-481.

The University of Chicago 